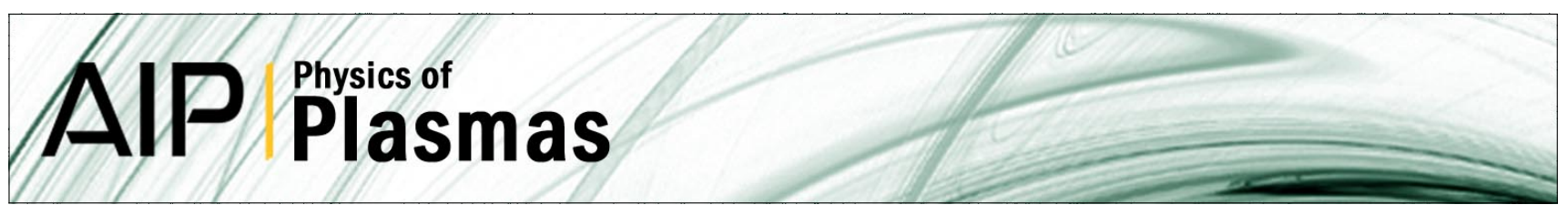

\title{
Gyrokinetic simulations of collisionless magnetic reconnection
}

B. N. Rogers, S. Kobayashi, P. Ricci, W. Dorland, J. Drake et al.

Citation: Phys. Plasmas 14, 092110 (2007); doi: 10.1063/1.2774003

View online: http://dx.doi.org/10.1063/1.2774003

View Table of Contents: http://pop.aip.org/resource/1/PHPAEN/v14/i9

Published by the American Institute of Physics.

\section{Related Articles}

Morphology and dynamics of three interacting kink-unstable flux ropes in a laboratory magnetoplasma Phys. Plasmas 19, 102102 (2012)

Magnetic reconnection in space

Phys. Plasmas 19, 092902 (2012)

Spectroscopic observation of simultaneous bi-directional reconnection outflows in a laboratory plasma Phys. Plasmas 19, 080704 (2012)

The role of compressibility in energy release by magnetic reconnection

Phys. Plasmas 19, 082109 (2012)

Design of a dual sensor probe array for internal field measurement in Versatile Experiment Spherical Torus Rev. Sci. Instrum. 83, 10D721 (2012)

\section{Additional information on Phys. Plasmas}

Journal Homepage: http://pop.aip.org/

Journal Information: http://pop.aip.org/about/about_the_journal

Top downloads: http://pop.aip.org/features/most_downloaded

Information for Authors: http://pop.aip.org/authors

\section{ADVERTISEMENT}

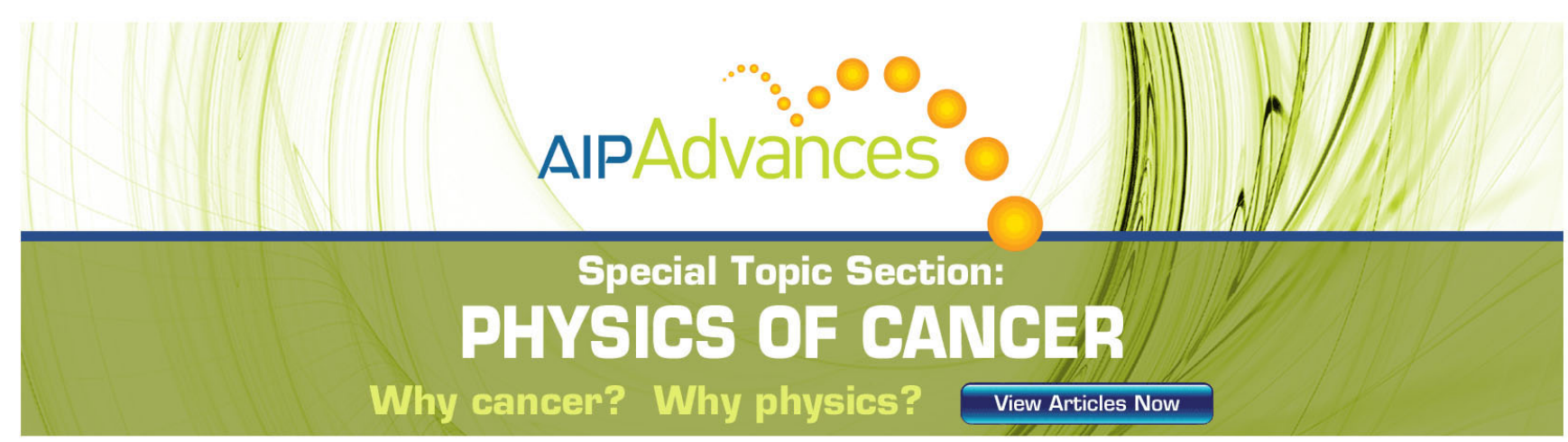




\title{
Gyrokinetic simulations of collisionless magnetic reconnection
}

\author{
B. N. Rogers ${ }^{\text {a) }}$ and S. Kobayashi \\ Department of Physics and Astronomy, Dartmouth College, Hanover, New Hampshire 03755, USA \\ P. Ricci \\ Centre de Recherches en Physique des Plasmas, École Polytechnique Fédérale de Lausanne, \\ Association EURATOM-Confédération Suisse, CH-1015 Lausanne, Switzerland \\ W. Dorland, J. Drake, and T. Tatsuno \\ University of Maryland, College Park, Maryland 20742, USA
}

(Received 3 July 2007; accepted 27 July 2007; published online 20 September 2007)

\begin{abstract}
Linear and nonlinear gyrokinetic simulations of collisionless magnetic reconnection in the presence of a strong guide field are presented. A periodic slab system is considered with a sinusoidally varying reconnecting magnetic field component. The linear growth rates of the tearing mode in both the large and small $\Delta^{\prime}$ regimes are compared to kinetic and fluid theory calculations. In the nonlinear regime, focusing on the limit of large $\Delta^{\prime}$, the nonlinear reconnection rates in the gyrokinetic simulations are found to be comparable to those obtained from a two-fluid model. In contrast to the fluid system, however, for $T_{i} \gg T_{e}$ and very small initial perturbation amplitudes, the reconnection in the gyrokinetic system saturates in the early nonlinear phase. This saturation can be overcome if the simulation is seeded initially with sufficient random noise. (C) 2007 American Institute of Physics. [DOI: 10.1063/1.2774003]
\end{abstract}

\section{INTRODUCTION}

We present linear and nonlinear gyrokinetic simulations of collisionless magnetic reconnection in the presence of a strong guide field. Our simulations are based on the GS2 code ${ }^{1,2}$ which time evolves the nonlinear electromagnetic gyrokinetic equations $^{3}$ for both electrons and ions. These equations, together with Maxwell's equations, govern the evolution of the electron and ion distribution functions (depending here on 2 spatial and 2 velocity-space coordinates) and the self-consistent electric and magnetic fields. A summary of the full system of equations is given in Appendix A.

The GS2 formalism is based on the reduced magnetohydrodynamic (MHD) ordering (see, e.g., Ref. 4), in which the deviations of the absolute levels of the density, temperatures, and the magnetic field (for example) are assumed to be small over the region of interest, though deviations in the gradients of these quantities can be comparable to or larger than the equilibrium values. Such an ordering is appropriate to the study of strong guide field reconnection, in which the component of the magnetic field that reconnects is small compared to the total magnetic field strength. The magnetic field and plasma profile variations associated with reconnection in this case, even in the nonlinear phase, are small compared to the unperturbed background values. Similarly, the time variations associated with reconnection in the large guide-field regime are slow compared to the ion gyroperiod. These properties are both necessary for the gyrokinetic approach, in which one of the three velocity-space coordinates (present in a full Vlasov treatment, for example) is eliminated from the system by a gyroangle average. This is computationally an advantage relative to full-particle simulations because it

\footnotetext{
${ }^{a)}$ Electronic mail: barrett.rogers@ dartmouth.edu
}

avoids the necessity to time-resolve the gyroperiod of the particles, which in the strong guide field limit is much faster than the time scale on which the modes of interest evolve. A similar computational gain is made in GS2 by enforcing total pressure balance, which eliminates compressional magnetosonic waves from the system while preserving the shearAlfvén dynamics that are essential to reconnection.

We consider a collisionless periodic slab geometry with a sinusoidally varying reconnecting magnetic field component $B_{y}(x) \propto \sin \left(k_{x} x\right)$. Depending on the dominant wavelength of the tearing mode under consideration (i.e., $k_{y}$ $=2 \pi / L_{y}$, where $L_{y}$ is the length of the system in the $y$-direction), this system allows us to explore reconnection in either the limits of large $\Delta^{\prime}$ (relevant to the $m=1$ mode in tokamaks, for example, or the GEM Reconnection Challenge Project ${ }^{5}$ ) or small $\Delta^{\prime}$ (relevant to tokamak tearing modes with $m>1$ ). We focus on a parameter regime of interest to magnetic confinement fusion experiments in which $\rho_{s}>d_{e}$, where $\rho_{s}=c_{s} / \Omega_{c i}$ is the ion sound Larmor radius, $d_{e}=c / \omega_{p e}$ is the collisionless electron skin depth, $\omega_{p e}^{2}=4 \pi n e^{2} / m_{e}$, $c_{s}^{2}=\left(T_{i}+T_{e}\right) / m_{i}, \Omega_{c i}=e B /\left(m_{i} c\right)$. Both the linear and nonlinear regimes are considered. In the linear case, we compare the gyrokinetic growth rates to analytical and numerical calculations based on kinetic and two-fluid models. Although reasonably good agreement between the two is found in some cases (small $T_{i} / T_{e}$ and low $\beta$ ), some of the parameters explored here $\left(T_{i} \geqslant T_{e}\right.$ and $\left.\beta \sim 1\right)$ fall in a regime in which existing analytic calculations are not clearly reliable. A rigorous treatment of these cases would seem to require the full gyrokinetic model and include, among other things, both electron and ion finite Larmor radius (FLR) effects. Although such an analysis is beyond the scope of the present article, derivations of some of the analytic results to which we com- 
pare are given in Appendix B, and the problems with these calculations are discussed.

In the nonlinear regime, we focus on a large- $\Delta^{\prime}$ case that corresponds approximately to the fastest-growing linear mode evolved into the nonlinear regime. We find the reconnection rates in the gyrokinetic system are comparable to those obtained from simulations based on a two-fluid model. An exception arises for $T_{i} \gg T_{e}$ and very small initial perturbation amplitudes, in which the nonlinear growth of the mode in the gyrokinetic system saturates in the early nonlinear phase (island widths comparable to $\rho_{i}$ ). This saturation can be overcome if small-amplitude random noise is added to the initial configuration.

The results discussed in this paper build on a number of other gyrokinetic studies of linear and nonlinear reconnection. Reference 6 applies gyrokinetic particle simulations to the study small-scale magnetic islands (widths comparable to the electron skin depth $\left.d_{e}=c / \omega_{p e}\right)$ in the presence of a narrow $\left(\right.$ about $10 d_{e}$ ) current sheet. References 7 and 8 explore reconnection in the small $\Delta^{\prime}$ case with gyrokinetic ions and drift-kinetic electrons. In larger box sizes $\left(\sim 64 \rho_{i}\right)$ an instability with odd parity was found to dominate the system, in contrast to the expected even parity of the tearing mode. In the large $\Delta^{\prime}$ nonlinear simulations described here, such oddparity modes have not been observed.

This paper is organized as follows: In Sec. II we describe the initial equilibrium and numerical simulation model. In Sec. III we compare the GS2 simulation results to linear theory calculations. We turn to the nonlinear regime in Sec. IV, in which we address the reconnection rate and saturation behavior. Our main conclusions are summarized in Sec. V. The gyrokinetic equations used in the simulations are described in Appendix A, and the derivations of various linear theory results are given in Appendix B.

\section{INITIAL EQUILIBRIUM AND SIMULATION MODEL}

The initial, unperturbed equilibrium state we consider is given by

$\vec{B}=B_{z 0} \hat{z}+B_{y 0}(x) \hat{y}, \quad B_{y 0}(x)=B_{y 0 \max } \sin \left(k_{x} x\right), \quad B_{z 0} \gg B_{y 0}$,

$$
n=n_{0}=\text { const }, \quad T_{i}=T_{i 0}=\text { const }, \quad T_{e}=T_{e 0}=\text { const. }
$$

For most of the numerical simulations discussed in this work we take $k_{x} \rho_{s e}=0.2$ (equivalent to a box size of $L_{x}=2 \pi / k_{x}$ $=10 \pi \rho_{\text {se }}$ ), where

$$
\begin{aligned}
& \rho_{s e}=\frac{c_{s e}}{\Omega_{c i}}=\sqrt{\frac{T_{e}}{m_{i}}} \frac{m_{i} c}{e B_{z 0}}=\sqrt{\frac{\beta_{e}}{2}} d_{i}, \\
& d_{i}^{2}=\frac{c^{2}}{\omega_{p i}^{2}}=\frac{c^{2} m_{i}}{4 \pi n_{0} e^{2}}, \\
& \beta_{e}=\frac{8 \pi n_{0} T_{e}}{B_{z 0}^{2}} .
\end{aligned}
$$

Other definitions that will be used later include

$$
\begin{aligned}
& \rho_{s}^{2}=\left(1+T_{i} / T_{e}\right) \rho_{s e}^{2}=\frac{\beta}{2} d_{i}^{2}, \\
& \beta=\frac{8 \pi n_{0}\left(T_{i}+T_{e}\right)}{B_{z 0}^{2}}, \\
& d_{e}^{2}=\frac{c^{2}}{\omega_{p e}^{2}}=\frac{m_{e}}{m_{i}} d_{i}^{2} .
\end{aligned}
$$

Note that $\rho_{s e}$ is the ion sound Larmor radius based on $T_{e}$ while $\rho_{s}$ is based on $T_{e}+T_{i}$.

A summary of the gyrokinetic and Maxwell equations solved by the GS2 code is given in Appendix A. The temporal discretization in GS2 to solve the linear terms in these equations is implicit and second order accurate in time. Nonlinearities are evaluated using an explicit Adams-Bashforth time-stepping scheme. In the $x$ and $y$ directions (the 2D plane of reconnection simulated here) a dealiased pseudospectral algorithm is used. In the $x$-direction the simulations include $n_{x}$ Fourrier modes with $64 \leqslant n_{x} \leqslant 8192$, depending on parameters. In the $y$ direction the nonlinear runs have a box size $L_{y}=2.5 L_{x}=25 \pi \rho_{s e}$ (corresponding to $k_{y, \min } \rho_{s e}=0.08$ ) and 64 $\leqslant n_{y} \leqslant 256$. The grid points in velocity space are chosen using Gaussian integration rules. Typical runs include 16 grid points in the particle (ion or electron) energies $\epsilon_{\alpha}=m_{\alpha} v_{\alpha}^{2} / 2$ in the velocity range $0 \leqslant v_{\alpha} \leqslant 6 v_{\text {th } \alpha}$, and 10 points in $\xi_{\alpha}=v_{\| \alpha} / v_{\alpha}$. The simulations within this range appear to be well converged and show little dependence on the velocityspace resolution. As discussed later, to facilitate parameter scans using modest numerical resources, an artificial electron to ion mass ratio is used, either $m_{e} / m_{i}=1 / 25$ or $m_{e} / m_{i}$ $=1 / 100$.

\section{LINEAR REGIME}

The simulations described in this section address two main cases

$$
\text { Case I: } \begin{aligned}
k_{x} \rho_{s e} & =0.2, \quad m_{i} / m_{e}=100, \quad \beta_{e}=0.2, \\
T_{i} / T_{e} & =\left(2.0 \times 10^{-4}, 5\right),
\end{aligned}
$$

Case II: $k_{x} \rho_{s e}=0.2, \quad m_{i} / m_{e}=25, \quad \beta_{e}=0.3$,

$$
T_{i} / T_{e}=\left(2.0 \times 10^{-5}, 5\right) .
$$

The simulation results in cases I and II are shown in Figs. 1 and 2, respectively. The black "+" symbols denote the GS2 values for very small $T_{i} / T_{e} \ll 1$ and the blue triangles represent $T_{i} / T_{e}=5$. Simulations at $T_{i} / T_{e}=1$ (not shown) are intermediate between the two, indicating the gyrokinetic growth rates (though not the eigenmode structure, as we show later) are only very weakly dependent on $T_{i} / T_{e}$. Since $\beta_{e}$ is held fixed in both cases, this is equivalent to an insensitivity to either the ion $\beta_{i}$ or the total plasma $\beta$, which for the parameters of case II, for example, varies from $\beta=0.3$ for $T_{i} / T_{e}$ $\simeq 0$ to $\beta=1.8$ for $T_{i} / T_{e}=5$. Both cases I and II satisfy the inequality $\rho_{s}^{2} \gg d_{e}^{2}$ (equivalent to $\beta \gg 2 m_{e} / m_{i}$ ), which, aside from being relevant to fusion devices, is required to allow comparison to some analytic calculations discussed below. 


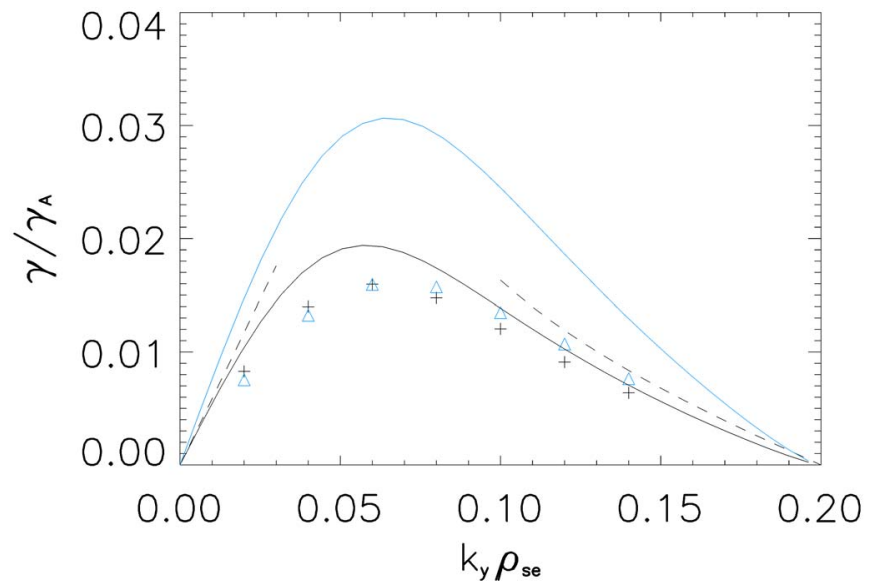

FIG. 1. (Color online) Linear growth rates $\gamma / \gamma_{A}$ vs $k_{y} \rho_{s e}$ for case I with $\gamma_{A}$ given by Eq. (10) and $\rho_{s e}=c_{s e} / \Omega_{c i}$. The "+" are GS2 values for $T_{i} / T_{e} \ll 1$, triangles $T_{i} / T_{e}=5$. The left and right dashed lines are Eqs. (10) and (11), respectively, with $T_{i}=0$. The solid lines are the numerical solution of a reduced four-field fluid model for $T_{i}=0$ (lower) and $T_{i}=5 T_{e}$ (upper).

These analytic results are shown in Figs. 1 and 2 as the dashed and solid lines.

Numerical convergence tests have been carried out across the full range of $k_{y}$. The spatial resolution requirements in the $x$-direction for convergence of the growth rates in the smaller- $k_{y}$ regime are modest $\left(n_{x} \sim 64\right.$ for $k_{y} \rho_{s e}=0.08$ and $\left.m_{i} / m_{e}=25\right)$ but increase strongly as $k_{y}$ is increased $\left(n_{x} \sim 8192\right.$ for $k_{y} \rho_{s e}=0.16$ and $\left.m_{i} / m_{e}=100\right)$. Such fine resolution levels in turn lead to severe time-step restrictions. Consistent with the observed trend in the resolution, the linear theory calculations discussed later suggest the tearing layer becomes narrower as $k_{y}$ is increased. The number of modes required at higher $k_{y}$ exceeds what one might expect from simple estimates, however, and is still under study.

The $k_{y}$ dependence of $\gamma$ seen in Figs. 1 and 2 is governed by the tearing mode stability parameter $\Delta^{\prime}{ }^{9}$ This parameter may be defined in terms of $\widetilde{B}_{x}$, the $x$-component of the magnetic field perturbation in the ideal regions outside the

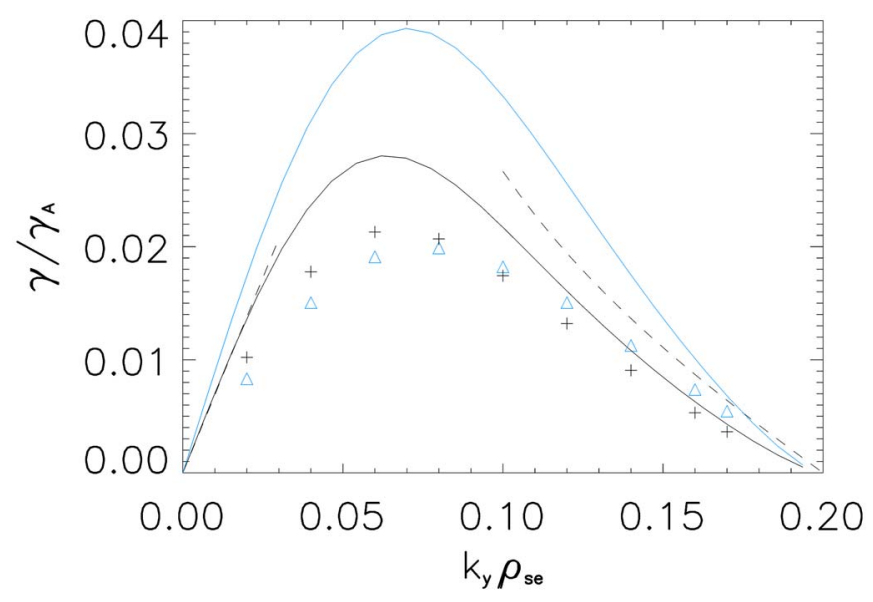

FIG. 2. (Color online) Linear growth rates for case II with the same notation as Fig. 1 .

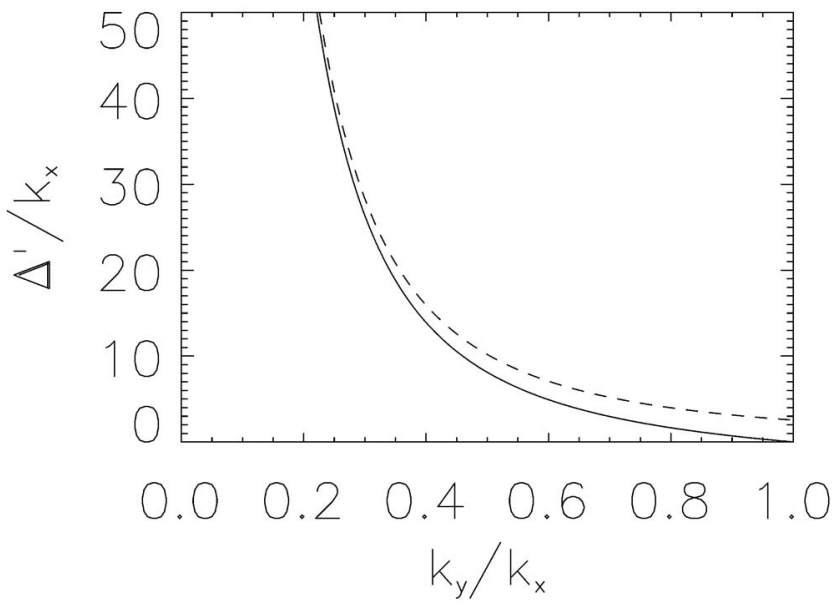

FIG. 3. $\Delta^{\prime} / k_{x}$ given by Eq. (8) vs $k_{y} / k_{x}$.

tearing layer. In our system, considering the layer at $x=0$ (see, e.g., Ref. 10),

$$
\widetilde{B}_{x} \propto \cos \left[D\left(k_{x}|x|-\pi / 2\right)\right] \sin \left(k_{y} y\right), \quad D \equiv \sqrt{1-\left(k_{y} / k_{x}\right)^{2}}
$$

where $k_{y}$ is the wavenumber of the linear mode of interest, and

$$
\Delta^{\prime}=\left[\frac{\partial_{x} \widetilde{B}_{x}}{\widetilde{B}_{x}}\right]_{x=0-}^{x=0+}=2 k_{x} D \tan (\pi D / 2) .
$$

A plot of $\Delta^{\prime} / k_{x}$ is shown in Fig. 3 as a function of $k_{y} / k_{x}$ (solid curve). Consistent with Figs. 1 and 2 tearing modes are unstable in the regime $k_{y}<k_{x}$ (here, $\left.k_{y} \rho_{s e}<0.2\right)$ in which $\Delta^{\prime}>0$. For $k_{y} \ll k_{x}, \Delta^{\prime} / k_{x} \simeq 8 k_{x}^{2} /\left(\pi k_{y}^{2}\right) \gg 1$ (dashed curve). The small- $\Delta^{\prime}$ regime lies to the right of the peak $\gamma$ values in Figs. 1 and 2; in this regime $\Delta^{\prime}$ is sufficiently small so that the constant- $\psi$ approximation is valid across the tearing layer. The large- $\Delta^{\prime}$ regime includes the peak growth rates as well as the regions to the left of the peaks in which the growth rates increase (at these parameters) linearly with $k_{y}$. The $k_{y}$ value of the peak growth rate and the corresponding value of $\Delta^{\prime}$ can be estimated, for example, by balancing the expression for $\gamma$ that applies to the left of the peak [in the large- $\Delta^{\prime}$ regime; see Eq. (10)] with the expression for $\gamma$ that applies to the right of the peak [in the small- $\Delta^{\prime}$ regime; see Eq. (11)]. This yields

$$
\Delta_{\max }^{\prime} \simeq 2^{1 / 3} \pi^{2 / 3} \rho_{s}^{-1 / 3} d_{e}^{-2 / 3}
$$

For the parameters of cases I and II this reduces to $\Delta_{\max }^{\prime} \simeq 28$ and $\Delta_{\max }^{\prime} \simeq 20$, respectively, or (with Fig. 3 and $\left.k_{x} \rho_{s e}=0.2\right), k_{y} \rho_{s e} \simeq 0.06$ for either case, consistent with the location of the peaks in Figs. 1 and 2. 


\section{A. Large $\Delta^{\prime}$}

In the regime $\rho_{s}>d_{e}$ under consideration, various analytic results have been obtained in the limits of both large and small $\Delta^{\prime}$. At large $\Delta^{\prime}$, the analytic results typically apply in the regions to the left of the peak growth rates in Figs. 1 and 2 where $\gamma \propto k_{y}$. The expression for $\gamma$ obtained in Ref. 10, for example, is given by

$$
\begin{aligned}
& \gamma=\left(\frac{2}{\pi}\right)^{1 / 3} k_{y} d_{e}^{1 / 3} \rho_{s}^{2 / 3} \gamma_{A}, \\
& \gamma_{A}=\frac{1}{\sqrt{4 \pi n_{0} m_{i}}}\left[\frac{d B_{y 0}}{d x}\right]_{x=0}=\frac{k_{x} B_{y 0 \max }}{\sqrt{4 \pi n_{0} m_{i}}} .
\end{aligned}
$$

This result was obtained in Ref. 10 using an isothermal fluid electron model and an ion model that includes leading-order FLR effects for arbitrary $T_{i} / T_{e}$ but ignores sound-wave coupling under the assumption $\gamma \gg k_{\|} c_{s}$. [A similar result, smaller than Eq. (10) by a factor of $\sqrt{2} / \pi^{1 / 6} \simeq 1.17$, was also obtained in Ref. 11 for $T_{i} \ll T_{e}$.] For convenience, a derivation of Eq. (10) is given in Appendix B, where we also show that a drift-kinetic electron treatment (along with a Padé approximation to the electron plasma dispersion function) gives the same result as the isothermal fluid electron model used in Ref. 10. In the case of $T_{i} \ll T_{e}$ Eq. (10), shown as dashed lines on the left-hand sides of Figs. 1 and 2, agrees reasonably well with the gyrokinetic results. As we discuss in more detail later, for small $k_{y}$ Eq. (10) is also approximately equivalent to an isothermal two-fluid model result shown as the upper $\left(T_{i} / T_{e}=5\right)$ and lower $\left(T_{i} / T_{e}=0\right)$ solid curves. These curves were obtained by solving numerically a set of four coupled reduced fluid equations described in Ref. 12, which go beyond Eq. (10) to allow for ion sound wave coupling and arbitrary $\beta$, but are formally valid only for $T_{i} \ll T_{e}$. These curves are also essentially indistinguishable from a numerical solution (not shown) of the full, nonreduced two-fluid model that is discussed in Sec. IV. To generate the upper $\left(T_{i} / T_{e}=5\right)$ solid curves in the figures, we have incorporated finite $T_{i}$ effects into the fluid model of Ref. 12 by the replacement $T_{e} \rightarrow T_{e}+T_{i}$. This is equivalent to assuming isothermal ion and electron equations of state and is consistent with the $T_{i} / T_{e}$ dependencies of Eqs. (10) and (11). It is not, however, consistent with the gyrokinetic growth rates, which for our parameters show a much weaker dependence on $T_{i} / T_{e}$. Indeed, at large $\Delta^{\prime}$ the analytic and fluid model growth rates increase with $T_{i} / T_{e}$ while the gyrokinetic results display the opposite trend.

\section{B. Small $\Delta^{\prime}$}

Turning now to the case of small $\Delta^{\prime}$, the dashed lines on the right-hand sides of Figs. 1 and 2 show the following asymptotic result of Refs. 10 and 13 evaluated at $T_{i}=0$ :

$$
\gamma=\frac{1}{\pi} k_{y} \rho_{s} \gamma_{A} d_{e} \Delta^{\prime}
$$

Like Eq. (10), for convenience this expression is derived in Appendix B from a kinetic ion treatment and either an isothermal drift-kinetic or fluid electron model. A similar result was also obtained in Refs. 14 and 15, aside from the replacement $\rho_{s} \rightarrow \rho_{s e}$ and an overall factor of $\sqrt{\pi} / 2 \simeq 1.13$. The GS2 results indicate that $\gamma$ increases much more weakly with $T_{i} / T_{e}$ than either Eq. (11) or the two-fluid model. We note that earlier versions of the two fluid model ${ }^{16,17}$ that neglected the electron gyroviscous terms in the electron momentum equation show much poorer agreement with the gyrokinetic simulations in the collisionless small- $\Delta^{\prime}$ limit.

\section{Limitations of the analytic and fluid model results}

The analytic and fluid model results shown in Figs. 1 and 2 rely on some simplifying assumptions that are not clearly valid in our parameter regime. As we now explain, a simple isothermal or adiabatic equation of state cannot be strictly correct in both the innermost electron layer where $\gamma \gtrsim k_{\|} v_{\text {the }}$ and the outermost zone in which $\gamma \ll k_{\|} v_{\text {the }}$ [note $k_{\|}=k_{y} B_{y}(x) / B \simeq k_{\|}^{\prime} x$ is $x$-dependent within the tearing layer; see Eq. (B3)]. Similarly, the nongyrokinetic calculations neglect electron FLR effects, the electron polarization drift, and ion sound wave coupling (the fluid model includes the latter but only in the zero-ion-FLR limit); all effects that, for $T_{i} \sim T_{e}$, become non-negligible at essentially the same point. Since ion FLR and finite- $\beta$ effects at $T_{i}=5 T_{e}$ are also nonnegligible, reliable analytic calculations in our higher- $T_{i}$ parameter regime are thus a formidable challenge and have yet to be carried out.

Electron FLR effects are potentially important because the electron motion [see, e.g., the expression for the current density given by Eq. (B15)], for either large or small $\Delta^{\prime}$, varies on the scale length

$$
\delta=\frac{\gamma}{k_{\|}^{\prime} v_{\mathrm{th}}}=\frac{\gamma d_{e}}{k_{y} \rho_{s} \gamma_{A}}, \quad v_{\mathrm{th}}=\sqrt{\frac{T_{e}+T_{i}}{m_{e}}} .
$$

The neglect of electron FLR effects requires that this scale, which at large $\Delta^{\prime}$ is a weakly decreasing function of $T_{i} / T_{e}$, exceed $\rho_{e}$; a condition that for the parameters of cases I and II and large $\Delta^{\prime}$ is marginally satisfied at $T_{i}=0$ but is violated at $T_{i} / T_{e}=5$. Similarly, at $T_{i} / T_{e}>1, E_{\|}$and $\phi$ also exhibits fine-scale structure for $x \sim \delta$ [see Eq. (B21)]:

$$
E_{\|} \simeq E_{\|}(0)\left(\frac{\delta^{2}+\frac{T_{e}}{T_{e}+T_{i}} x^{2}}{\delta^{2}+x^{2}}\right) .
$$

This expression, similar to the behavior noted in Ref. 18, predicts that $E_{\|}$for $T_{i} / T_{e}>1$ drops by a factor of $T_{e} /\left(T_{e}\right.$ $+T_{i}$ ) on the $\delta$ scale. For larger $x \gg \delta\left[\right.$ see Eq. (B22)] $E_{\|}$then decays to zero on the $\rho_{s}$ scale. The consistency of this with the simulations can be seen in Fig. 4, which shows $E_{\|} / E_{\|}(0)$ in the linear regime for the case II parameters at $k_{y} \rho_{s e}$ $=0.05$ (large $\left.\Delta^{\prime}\right)$. The width of the narrow peak at $x=0$ for $T_{i} / T_{e}=5$ (solid line) is comparable to $\rho_{e}$ and is broader than $\delta$, consistent with the expectation that $\rho_{e}$ effects are becoming important. At $T_{i} \ll T_{e}$ (dashed line), such fine-scale structure is absent as expected from Eq. (13).

A related effect, which only becomes important at finite $T_{i} / T_{e}$, is the electron polarization drift [see Eqs. (B30) or (B33)], 


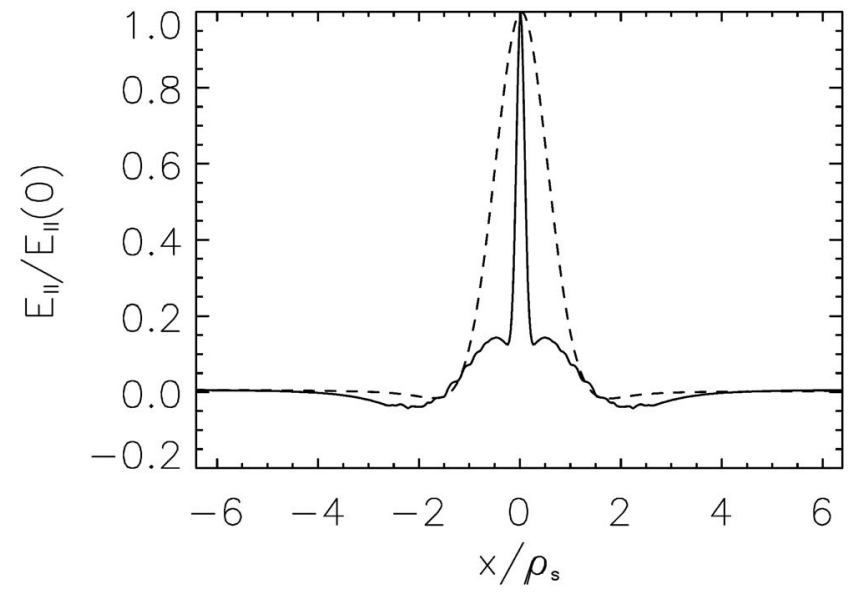

FIG. 4. $E_{\|} / E_{\|}(0)$ for case II with $k_{y} \rho_{s e}=0.05$ and $T_{i} / T_{e}=5$ (solid), $T_{i} / T_{e}$ $=2 \times 10^{-5}$ (dashed). Sub- $\rho_{s}$ structure appears only for $T_{i} / T_{e} \sim 1$ or larger.

$$
\tilde{n}_{e, \mathrm{pol}} \sim-\frac{n_{0} e}{T_{e}} \rho_{e}^{2} \nabla^{2} \phi
$$

When $k_{\perp} \rho_{i} \ll 1$ and the ions are magnetized, the contribution of $\tilde{n}_{e, \text { pol }}$ is negligible compared to the ion polarization drift. At finite $T_{i}$, however, the ion response for $x \sim \delta \ll \rho_{i}$ becomes adiabatic [see Eq. (B1), neglecting $\Gamma_{0}$ ],

$$
\tilde{n}_{i} \sim-\frac{n_{0} e}{T_{i}} \phi
$$

Estimating $\nabla^{2} \phi \sim \phi / \delta^{2}$, the contribution of Eq. (14) is found to be negligible compared to Eq. (15) when $\left(T_{i} / T_{e}\right) \rho_{e}^{2} \ll \delta^{2}$. This condition may be combined with the electron FLR restriction $\rho_{e}^{2} \ll \delta^{2}$ to yield

$$
\frac{\left(1+T_{i} / T_{e}\right) \rho_{e}^{2}}{\delta^{2}}=\frac{\beta}{2} \frac{k_{y}^{2} \rho_{s}^{2} \gamma_{A}^{2}}{\gamma^{2}} \ll 1 .
$$

Again, at large $\Delta^{\prime}$, this condition for the case I and II parameters is marginally satisfied at $T_{i} \ll T_{e}$ but is violated for $T_{i} / T_{e}=5$. With the expression for $\gamma$ given by Eq. (10), it may also be written as $\beta / 2<\left(m_{e} / m_{i}\right)^{1 / 4}$, a condition that was also emphasized in Refs. 16 and 17. Due to the $k_{y}^{2}$ factor in the numerator, Eq. (16) is even more difficult to satisfy at small $\Delta^{\prime}\left(\right.$ large $\left.k_{y}\right)$ than at large $\Delta^{\prime}\left(\right.$ small $\left.k_{y}\right)$. Thus, even for $T_{i}$ $\ll T_{e}$, we would expect electron FLR effects - absent in Eq. (11) and the two-fluid model-to play an even greater role.

Two other effects absent from Eqs. (10) and (11), (though not the two-fluid curves) are contributions from $\widetilde{B}_{\|}$, which become potentially significant for $T_{i}=5 T_{e}$ where $\beta \sim 1$, as well as ion sound wave coupling, which may be neglected only if $\gamma^{2} \gg k_{\|}^{2} c_{s}^{2}$ for $x \lesssim \rho_{s}$ [the outer envelope of the ion response, see Eq. (B18)]. Given $k_{\|}=k_{\|}^{\prime} x$ this condition may be written as $\gamma^{2} \gg k_{\|}^{\prime 2} \rho_{s}^{2} c_{s}^{2}$ or, since $\rho_{s}^{2} c_{s}^{2}=(1$ $\left.+T_{i} / T_{e}\right) \rho_{e}^{2} v_{\mathrm{th}}^{2}, \gamma^{2} \gg k_{\|}^{\prime 2} v_{\mathrm{th}}^{2}\left(1+T_{i} / T_{e}\right) \rho_{e}^{2}$. In the latter form, recalling that $\delta^{2}=\gamma^{2} /\left(k_{\|}^{\prime 2} v_{\text {th }}^{2}\right)$, one sees that this condition is exactly the same as Eq. (16). The proper account of ion sound wave coupling for $T_{i} \sim T_{e}$ is difficult because ion FLR effects are not negligible in this case, since $\rho_{s}$ (the main scale-length of the ion motion) is comparable to $\rho_{i}$.
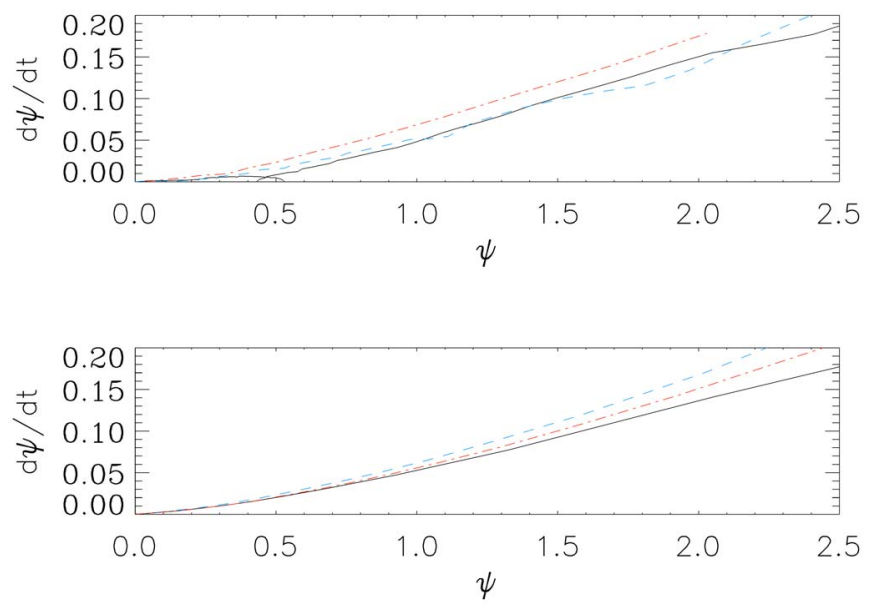

FIG. 5. (Color online) Gyrokinetic (upper panel) and two-fluid (lower panel) nonlinear reconnection rates for case II vs $\psi$, the reconnected flux inside the island, for $T_{i} \ll T_{e}$ (solid line), $T_{i}=T_{e}$ (dotted-dashed), $T_{i}=5 T_{e}$ (dashed).

In the case of the perpendicular ion dynamics, the importance of ion FLR effects has long been appreciated and was first studied in detail in Ref. 10. This work, like the calculations presented in Appendix B, is based on a Padé approximation of the perpendicular kinetic ion response that is leading-order accurate in the limits of both $k_{\perp} \rho_{i} \gg 1$ and $k_{\perp} \rho_{i} \rightarrow 0$. In view of the role of ion FLR effects in these calculations, it is perhaps surprising that the resulting dispersion relation [Eq. (B19)] for arbitrary $T_{i} / T_{e}$ may also be obtained from various two-fluid calculations ${ }^{12,16,17,19,20}$ in the limit that isothermal electron and ion fluid equations of state are used. The reason for this agreement, as we show in Appendix $\mathrm{B}$, is that the Padé approximation to the perpendicular kinetic ion response is exactly reproduced by the usual lowfrequency, reduced-MHD-type approach provided that the ion diamagnetic drift is properly included. The numerical solution to the four coupled reduced two-fluid equations discussed in Ref. 12, shown in Figs. 1 and 2 as the upper $\left(T_{i} / T_{e}=5\right)$ and lower $\left(T_{i}=0\right)$ solid lines, go beyond Eqs. (10) and (11) to include in the zero-FLR limit ion-sound-wave coupling and finite $\beta$ effects. While both of these effects are stabilizing, the fluid predictions for $T_{i}=5 T_{e}$ are still substantially larger than the GS2 values, indicating the kinetic effects discussed earlier are important.

\section{NONLINEAR REGIME}

Turning now to the nonlinear evolution, we again consider the parameters of case II discussed in Sec. III. As noted earlier the nonlinear runs have a box size $L_{y}=2.5 L_{x}$, corresponding to $k_{y \min } \rho_{s e}=0.08$. This minimum $k_{y}$ corresponds roughly to the fastest growing linear mode. Following the initialization of the run with small amplitude random noise, this longest-wavelenth mode dominates the system as the nonlinear phase is reached. Because this mode falls within in the large $\Delta^{\prime}$ regime, the simulations discussed here, aside from the guide field, are similar to those of the GEM Reconnection Challenge Project. ${ }^{5}$

Figure 5 shows the nonlinear reconnection rates $d \psi / d t$ 
for case II as a function of $\psi$, the reconnected flux inside the magnetic island. The upper panel shows the GS2 values for $T_{i} \ll T_{e}$ (solid line), $T_{i}=T_{e}$ (dotted-dashed), $T_{i}=5 T_{e}$ (dashed). The nonmonotonic feature in the curve for $T_{i} / T_{e} \ll 1$ near $\psi \simeq 0.5$ is due to the initial noise in the system, which at early times leads to competition between the dominant mode with $k_{y} \rho_{s e}=0.08$ and the next-order harmonic. Here $\psi$ is normalized to $B_{0, \max } d_{i}$ and $t$ to $\gamma_{A}$. At the final times shown in the figure the island widths are roughly half the box size $w$ $\sim L_{x} / 2$. In all three cases the reconnection continues until the islands span the entire box $\left(w \sim L_{x}\right)$, although the reconnection rates drop sharply at times just beyond those shown in the figure. The lower panel shows the analogous results obtained from a simple isothermal two-fluid simulation with the same parameters and a large guide field $\left(B_{z} \simeq 10 B_{x, \text { max }}\right)$. The generalized Ohm's law in this model includes the Hall terms and electron inertia. ${ }^{5}$ The reconnection rates in the gyrokinetic system are comparable to those in the fluid model and, like the linear gyrokinetic growth rates, exhibit little dependence on $T_{i} / T_{e}$. Numerical tests also show the contribution of $\widetilde{B}_{\|}$in the gyrokinetic equations (see Appendix A) to both the linear and nonlinear reconnection rates is small $(\lessgtr 5 \%)$, in contrast to what one might expect for the higher $T_{i}$ cases in which $\beta \sim \beta_{i} \sim 1$.

The fluid code used here was benchmarked in the GEM reconnection study ${ }^{5}$ against full-particle simulations and was found to produce similar rates of reconnection. Subsequent work with this same fluid code was carried out, e.g. in Ref. 21 , that explored the dependence of the reconnection rate on the guide field strength, the plasma $\beta$, and other parameters. In the strong guide field regime with $\rho_{s}>d_{e}$ relevant to our simulations, the reconnection rates were characterized by inflow velocities that scale roughly as a small fraction $(\sim 0.05)$ of the Alfvén speed based on the reconnecting component of the magnetic field just upstream of the reconnection region,

$$
V_{\text {in }} \sim \frac{\delta}{l} V_{\text {out }} \sim 0.05 V_{\text {out }}, \quad V_{\text {out }} \sim \frac{B_{y, \text { up }}}{\sqrt{4 \pi n m_{i}}} .
$$

Similar rates have also been observed ${ }^{22}$ in particle simulations of strong guide field reconnection with a realistic mass ratio, $m_{i} / m_{e}=1836$.

In the present system, the reconnecting component of the magnetic field near the reconnection layer is given by

$$
B_{y 0}(x)=B_{y 0 \max } \sin \left(k_{x} x\right) \simeq B_{y 0}^{\prime} x+\cdots, \quad B_{y 0}^{\prime}=k_{x} B_{y 0 \max }
$$

or in terms of the flux function $\left(B_{y}=\partial \psi / \partial x\right)$ :

$$
\psi \simeq \frac{1}{2} B_{y 0}^{\prime} x^{2} .
$$

As reconnection proceeds in the nonlinear phase, the flux surfaces upstream of the reconnection layer at $x=0$ are shifted toward the layer, causing an increase in the local values of $B_{y}$ and $\psi$. Denoting this time dependent shift in the $x$ direction by $\xi(t)$, the values of $B_{y}$ and $\psi$ just upstream of the layer at $x=0$ can be estimated by replacing $x \rightarrow \xi$ in the equilibrium profiles,

$$
B_{y, \text { up }} \simeq B_{y 0}^{\prime} \xi, \quad \psi \simeq \frac{1}{2} B_{y 0}^{\prime} \xi^{2} .
$$

From the expression for $\psi$ one finds

$$
\frac{1}{\psi} \frac{d \psi}{d t}=\frac{2}{\xi} \frac{d \xi}{d t} .
$$

An expression for $d \xi / d t$ may be obtained from the continuity equation as

$$
\frac{d \xi}{d t}=V_{\text {in }}=\frac{\delta}{l} V_{\text {out }}, \quad V_{\text {out }} \sim \frac{B_{y, \text { up }}}{\sqrt{4 \pi n m_{i}}}, \quad B_{y, \text { up }}=B_{y 0}^{\prime} \xi
$$

or equivalently,

$$
\frac{d \xi}{d t}=\frac{\delta}{l} \frac{B_{y 0}^{\prime}}{\sqrt{4 \pi n m_{i}}} \xi
$$

Equation (21) can thus be written as

$$
\frac{1}{\psi} \frac{d \psi}{d t}=\frac{2}{\xi} \frac{d \xi}{d t}=\frac{2 \delta}{l} \frac{B_{y 0}^{\prime}}{\sqrt{4 \pi n m_{i}}}=\frac{2 \delta}{l} k_{x} V_{A y} .
$$

Estimating $\delta / l \sim 0.05$ and normalizing the time units to $\gamma_{A}$ as in Fig. 5, this expression reduces to

$$
\frac{d \psi}{d t}=\frac{2 \delta}{l} \gamma_{A} \psi \simeq 0.1 \psi
$$

One would therefore expect the slopes of the curves in Fig. 5 at early times to be constant and roughly equal to 0.1 , which indeed they are. The constancy of the slope implies that the reconnected flux and magnetic island width grow exponentially with time.

Concerning the insensitivity of the gyrokinetic system to $T_{i} / T_{e}$, a caveat must be added regarding the level of noise that is initially imposed on the simulations. The solid curve in Fig. 6 shows the island width as a function of time in the $T_{i}=5 T_{e}$ simulation shown in Fig. 5 (top panel). The initial noise level in this simulation is small but finite, such that values of $w$ smaller than about $w \sim 0.01 \rho_{\text {se }}$ (the starting value of the solid curve) cannot be distinguished from the initial noise. The island width in this case does not saturate and reconnection continues until essentially all the flux in the

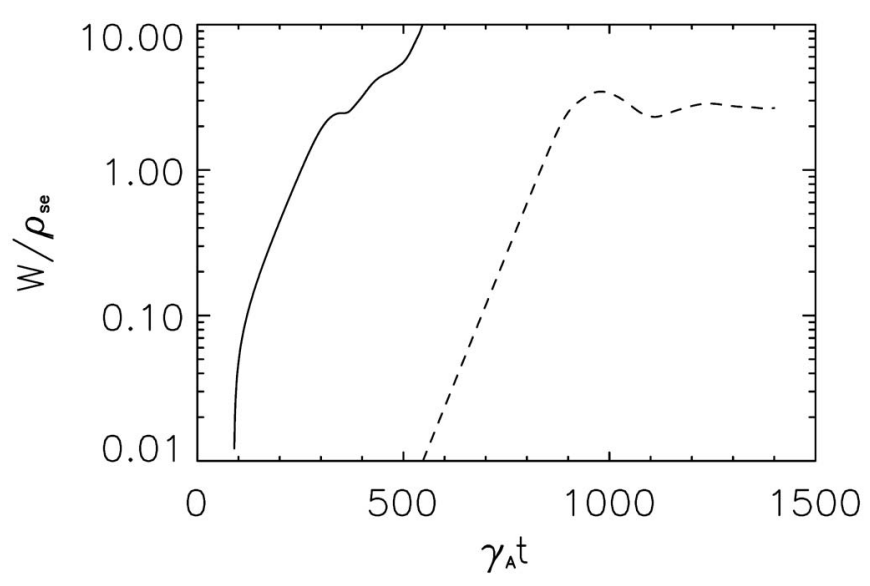

FIG. 6. Island widths $w / \rho_{s e}$ vs $\gamma_{A} t$ for $T_{i} / T_{e}=5$ with (solid) and without (dashed) added initial noise in the simulation. The nonlinear saturation is avoided for very weak levels of initial noise. 
system is reconnected. When the noise level is drastically reduced (dashed curve), however, the growth of the island halts at about $w \sim 2 \rho_{s e} \sim \rho_{i}$. Apparently the saturation mechanism, at least for these parameters, is fragile and easily destroyed by the presence of noise in the system - a feature that may explain why such saturation behavior has not been previously observed in particle simulations of similar largeguide-field systems (see, e.g., Ref. 23). Such early nonlinear saturation is not observed in the fluid simulation of the same system, or in the gyrokinetic simulations at smaller $T_{i} / T_{e}$.

Saturation behavior superficially similar to that observed here has been reported in one particle simulation study ${ }^{24}$ of reconnection in a high-beta system without a guide field. It was conjectured the saturation was due to the formation of electron temperature anisotropies $\left(T_{e \perp}<T_{e \|}\right)$, which are known to have a potentially strong stabilizing impact on the reconnection. In our system the initial plasma equilibrium is isotropic and in the strong guide-field limit the perturbations of the plasma temperatures, densities, and pressures are small (see, e.g., Ref. 25 for discussion). On the theoretical side, the saturation of tearing modes has been studied in both low and high $\beta$ plasmas (e.g., Refs. 26-28). Consistent with our findings, in Ref. 28 nonlinear saturation in the strong guide field regime is predicted when $w \sim \rho_{i}$ due to the interaction of the ions with the electrostatic field of the reconnecting mode (ion trapping). This study, however, considers systems in which an ambient pressure gradient (absent in the system considered here) leads to a real-frequency of the mode (also absent here) that is larger than the linear growth rate. Further study of this issue is needed, therefore, to determine if the physical mechanism described in that work is consistent with our simulations.

\section{CONCLUSION}

We have explored linear and nonlinear collisionless reconnection in the $\rho_{s}>d_{e}$ regime using gyrokinetic simulations. In the linear-theory limit, the gyrokinetic growth rates are compared to analytic calculations across a range of parameters, including small and large $\Delta^{\prime}$ and $T_{i} / T_{e}$. For the cases in which the analytic calculations of the growth rate are theoretically reliable for our parameters (low $\beta$ and small $T_{i} / T_{e}$ ), reasonably good agreement between the theory and simulations is found, while for some other cases $(\beta \sim 1$ and $T_{i} \gtrless T_{e}$ ), further analytic work is needed to provide a sound benchmark for the simulations. In particular, the insensitivity of the linear growth rates in the gyrokinetic model to $T_{i} / T_{e}$ and the plasma $\beta$, apparently due to the role played by FLR effects in the parameter regimes considered here, are not well reproduced by existing analytic calculations. In the nonlinear regime, the reconnection rates at large $\Delta^{\prime}$ were explored for various values of $T_{i} / T_{e}$, and found to be similar to those obtained from a two-fluid model. As in the linear case, the dependence of the nonlinear reconnection rates on $T_{i} / T_{e}$ is quite weak. An exception to this occurs at larger $T_{i} / T_{e}$ $\left(T_{i} / T_{e}=5\right)$ and very weak initial perturbations, in which the growth of the island width in the gyrokinetic system saturates at the $\rho_{i}$-scale. This saturation for our parameters may be overcome by adding small levels of initial noise to the simulations.

\section{ACKNOWLEDGMENTS}

This material is based upon work supported by NSF Grant No. 0238694, the Center for Multiscale Plasma Dynamics, and DOE Grant No. DE-FC02-04ER54784. P.R. is supported by a EURATOM Fusion fellowship.

\section{APPENDIX A: THE GS2 GYROKINETIC EQUATIONS}

The gyrokinetic equations, with species label $\alpha$, may be written as

$$
\begin{aligned}
\left(\frac{\partial}{\partial t}+\right. & \left.i \Omega_{d \alpha}+v_{\|} \nabla_{\|}\right) h_{\alpha}\left(\mathbf{k}_{\perp}\right)+\frac{c}{4 \pi^{2} B} \\
& \times \sum_{\mathbf{k}_{\perp}^{\prime}, \mathbf{k}_{\perp}^{\prime \prime}}\left[\mathbf{b} \cdot\left(\mathbf{k}_{\perp}^{\prime \prime} \times \mathbf{k}_{\perp}^{\prime}\right)\right] \cdot \chi\left(\mathbf{k}_{\perp}^{\prime}\right) h_{\alpha}\left(\mathbf{k}_{\perp}^{\prime \prime}\right) \delta_{\mathbf{k}_{\perp}^{\prime}+\mathbf{k}_{\perp}^{\prime \prime}, \mathbf{k}_{\perp}} \\
= & -\left(\frac{\partial}{\partial t}+i \Omega_{* \alpha}\right) \chi\left(\mathbf{k}_{\perp}\right) q_{\alpha} \frac{\partial F_{0 \alpha}}{\partial \epsilon} \\
& +\frac{1}{2 \pi} \int_{-\pi}^{\pi} \exp (-i L) C\left(f_{\alpha}\right) d \xi
\end{aligned}
$$

where $L=\left(\mathbf{v} \times \mathbf{b} \cdot \mathbf{k}_{\perp}\right) / \Omega_{c \alpha}, \xi$ is the gyroangle, $\epsilon=m_{\alpha} v^{2} / 2$ is the particle energy, $\mu=m_{\alpha} v_{\perp}^{2} / 2 B$ is the magnetic moment, $\chi$ is the generalized gyrokinetic potential

$$
\chi=J_{0}\left(\frac{k_{\perp} v_{\perp}}{\Omega_{c \alpha}}\right)\left(\phi-\frac{v_{\|}}{c} A_{\|}\right)+J_{1}\left(\frac{k_{\perp} v_{\perp}}{\Omega_{c \alpha}}\right) \frac{v_{\perp}}{k_{\perp} c} \delta B_{\|},
$$

$\phi$ is the electrostatic potential, $A_{\|}$is the parallel component of the vector potential, $\delta B_{\|}$is the perturbation of the parallel magnetic field, $\mathbf{b}$ is the magnetic-field unit vector, $\delta_{\mathbf{k}^{\prime \prime}, \mathbf{k}^{\prime}}=0$ for $\mathbf{k}^{\prime \prime} \neq \mathbf{k}^{\prime}$ and $\delta_{\mathbf{k}^{\prime \prime}, \mathbf{k}^{\prime}}=1$ for $\mathbf{k}^{\prime \prime}=\mathbf{k}^{\prime}, \Omega_{c \alpha}=q_{\alpha} B_{\theta} /\left(m_{\alpha} c\right)$, and $C\left(f_{\alpha}\right)$ is a collision operator (not relevant to the present work). The nonadiabatic part of the distribution function $h_{\alpha}=h_{\alpha}(\epsilon, \mu, t)$ is related to the total perturbed distribution function $f_{\alpha}=f_{\alpha}(\mathbf{v}, t)$ through $f_{\alpha}=q_{\alpha} \phi \partial F_{0 \alpha} / \partial \epsilon+\exp (i L) h_{\alpha}$, or $\left\langle f_{\alpha}\right\rangle_{\xi}=q_{\alpha} \phi \partial F_{0 \alpha} / \partial \epsilon+J_{0} h_{\alpha}$. The frequencies $\Omega_{d \alpha}$ and $\Omega_{*_{\alpha}}$ are defined as

$$
\begin{aligned}
\Omega_{* \alpha} & =\frac{\mathbf{b} \times \mathbf{k}_{\perp} \cdot \nabla F_{0 \alpha}}{m_{\alpha} \Omega_{c \alpha} \partial F_{0 \alpha} / \partial \epsilon}, \\
\Omega_{d \alpha} & =\mathbf{k}_{\perp} \cdot \mathbf{b} \times \frac{m_{\alpha} v_{\|}^{2} \mathbf{b} \cdot \nabla \mathbf{b}+\mu \nabla B}{m_{\alpha} \Omega_{c \alpha}} .
\end{aligned}
$$

The ion and electron "background" distribution functions $F_{0 \alpha}$ are assumed to be isotropic Maxwellians. The gyrokinetic equations are coupled to the Maxwell equations, which may be written as

$$
\begin{aligned}
& \sum_{\alpha} \int d^{3} v q_{\alpha}\left[q_{\alpha} \phi \frac{\partial F_{0}}{\partial \epsilon}+J_{0} h_{\alpha}\right]=0, \\
& \nabla_{\perp}^{2} A_{\|}=-\frac{4 \pi}{c} \sum_{\alpha} \int d^{3} v q_{\alpha} v_{\|} J_{0} h_{\alpha},
\end{aligned}
$$




$$
\delta B_{\|}=-\frac{4 \pi}{c} \sum_{\alpha} \int d^{3} v \frac{q_{\alpha} v_{\perp}}{k_{\perp}} J_{1} h_{\alpha} .
$$

\section{APPENDIX B: LINEAR THEORY}

Assuming $\gamma \gg k_{\|} c_{s}$, the ion density perturbation $\tilde{n_{i}}$ is given by (see, e.g., Ref. 10)

$$
\widetilde{n}_{i}=\frac{n_{0} e}{T_{i}}\left[\Gamma_{0}(b)-1\right] \phi, \quad \Gamma_{0}(b)=I_{0}(b) e^{-b}, \quad b=-\rho_{i}^{2} \nabla_{\perp}^{2},
$$

where $\rho_{i}=\sqrt{T_{i} / m_{i}} / \Omega_{c i}$ is the ion Larmor radius and $I_{0}$ is the modified Bessel function. The perturbed electron continuity equation gives

$$
\tilde{n}_{e} \simeq \frac{1}{\gamma e} \nabla_{\|} \widetilde{J}_{\|}=x \frac{c^{2} k_{\|}^{\prime 2}}{4 \pi e \gamma^{2}} A^{\prime \prime}, \quad A=\frac{\gamma}{i k_{\|}^{\prime} c} A_{z},
$$

where in the linear regime we have assumed

$$
\nabla_{\|}=i k_{\|}^{\prime} x, \quad k_{\|}^{\prime}=\frac{k_{y}}{B_{z 0}}\left[\frac{d B_{y 0}}{d x}\right]_{x=0}=\frac{k_{y} \gamma_{A}}{V_{A}}, \quad \tilde{J}_{\|} \simeq-\frac{c}{4 \pi} A_{z}^{\prime \prime} .
$$

The quasineutrality condition $\tilde{n_{i}}=\tilde{n_{e}}$ can thus be written as

$$
\frac{T_{e}}{T_{i}}\left[\Gamma_{0}-1\right] \phi=x \frac{k_{y}^{2} \rho_{s e}^{2} \gamma_{A}^{2}}{\gamma^{2}} A^{\prime \prime} .
$$

Finally, the generalized Ohm's law, assuming an isothermal electron response, is

$$
-\frac{m_{e} \gamma}{e} \widetilde{J}_{\|}=-\nabla_{\|}\left(T_{e} \tilde{n}_{e}\right)-n_{0} e E_{\|}
$$

or equivalently, using Eqs. (B2) and (B3)

$$
\begin{aligned}
& -\frac{E_{\|}}{i k_{\|}}=x \phi+A=\frac{k_{y}^{2} \rho_{s e}^{2} \gamma_{A}^{2}}{\gamma^{2}}\left(\delta_{e}^{2}+x^{2}\right) A^{\prime \prime}, \\
& \delta_{e}^{2}=\frac{\gamma^{2}}{k_{\|}^{\prime 2} v_{\text {the }}^{2}}, \\
& v_{\text {the }}^{2}=\frac{T_{e}}{m_{e}} .
\end{aligned}
$$

To make progress, we introduce the auxiliary variable $\phi_{i}$ defined as

$$
\frac{T_{e}}{T_{i}}\left[\Gamma_{0}-1\right] \phi \equiv \rho_{s e}^{2} \nabla_{\perp}^{2} \phi_{i}
$$

or equivalently,

$$
\phi=\frac{\rho_{i}^{2} \nabla_{\perp}^{2}}{\left[\Gamma_{0}-1\right]} \phi_{i} .
$$

We demonstrate below that $c \phi_{i} / B$ is the ion velocity stream function in two-fluid theory. Now making the well-known Padé approximation

$$
\Gamma_{0} \simeq \frac{1}{1-\rho_{i}^{2} \nabla_{\perp}^{2}}
$$

Eq. (B8) simply becomes

$$
\phi=\left(1-\rho_{i}^{2} \nabla_{\perp}^{2}\right) \phi_{i} .
$$

Eliminating $\phi$ in Eqs. (B4) and (B6) with Eqs. (B7) and (B10), respectively, one obtains

$$
\begin{aligned}
& \phi_{i}^{\prime \prime}=x \frac{k_{y}^{2} \gamma_{A}^{2}}{\gamma^{2}} A^{\prime \prime}=\frac{k_{y}^{2} \gamma_{A}^{2}}{\gamma^{2}} \partial_{x}\left[x^{2} \partial_{x}(A / x)\right], \\
& x \phi_{i}+A=\frac{k_{y}^{2} \rho_{s}^{2} \gamma_{A}^{2}}{\gamma^{2}}\left(\delta^{2}+x^{2}\right) A^{\prime \prime}, \quad \delta^{2}=\frac{\delta_{e}^{2}}{1+T_{i} / T_{e}} .
\end{aligned}
$$

Note that the contribution of the $\rho_{i}^{2} \partial_{x}^{2}$ terms arising from Eq. (B10) has simply led to the replacements $\delta_{e} \rightarrow \delta, \rho_{s e} \rightarrow \rho_{s}$ in Ohm's law, or equivalently, $T_{e} \rightarrow T_{e}+T_{i}$. The solutions for $\phi_{i}$ and $A$ for $x \rightarrow+\infty$ must be matched to the asymptotic forms

$$
\phi_{i} \simeq \phi_{\infty}=\text { const }, \quad A \simeq-\phi_{\infty}\left(x+2 / \Delta^{\prime}\right) .
$$

With this boundary condition, Eq. (B11) may be integrated from $x=0$ to $x=\infty$ to yield the constraint

$$
-\phi_{i}^{\prime}(0)=\frac{k_{y}^{2} \gamma_{A}^{2}}{\gamma^{2}}\left(\frac{2 \phi_{\infty}}{\Delta^{\prime}}+A(0)\right) \text {. }
$$

The constants in this expression may be related to each other from the solution to Eqs. (B11) and (B12). Assuming $\rho_{s}>d_{e}$, this solution, discussed for example in Ref. 20, turns out to have two scales: an inner scale $\delta$ that determines the width of the current profile (i.e., $A^{\prime \prime}$ ), and an outer scale $\rho_{s}$ that determines the behavior of $\phi_{i}$. On the inner scale, the $\phi_{i}$ term in Eq. (B12) can be neglected and one can take $A=A_{0}$ $+A_{1}+\cdots$, where $A_{0}=A(0)$ is a constant (that is, the constant $\psi$ approximation is valid), yielding

$$
A^{\prime \prime}=A_{1}^{\prime \prime} \simeq \Gamma^{2} \frac{A_{0}}{\left(\delta^{2}+x^{2}\right)}, \quad \Gamma=\frac{\gamma}{k_{y} \rho_{s} \gamma_{A}} .
$$

Integrating this expression and comparing it to Eq. (B13) for $x \gg \delta$ one obtains

$$
A^{\prime} \simeq \Gamma^{2} \frac{A_{0}}{\delta} \tan ^{-1}(x / \delta)=-\phi_{\infty} \frac{2}{\pi} \tan ^{-1}(x / \delta) .
$$

In the outer region $x \gg \delta$, one can neglect the $\delta^{2}$ term in Eq. (B12) and eliminate $A^{\prime \prime}$ with Eq. (B11), yielding

$$
x \phi_{i}+A=x \rho_{s}^{2} \phi_{i}^{\prime \prime} .
$$

Differentiating this equation with respect to $x$, using $A^{\prime} \simeq-\phi_{\infty}$, and noting $\phi_{i}(0)=0$, one finds the solution

$$
\phi_{i} \simeq \phi_{\infty}\left(1-e^{-x / \rho_{s}}\right) .
$$

Finally, returning to Eq. (B14) with this solution, and using [from Eq. (B16)] $A(0)=-2 \delta \phi_{\infty} /\left(\Gamma^{2} \pi\right)=-2 d_{e} \phi_{\infty} /(\Gamma \pi)$, one obtains the dispersion relation 


$$
\Gamma^{3}=\frac{\gamma^{3}}{k_{y}^{3} \rho_{s}^{3} \gamma_{A}^{3}}=\frac{2}{\pi} \frac{d_{e}}{\rho_{s}}\left(1-\frac{\Gamma \pi}{d_{e} \Delta^{\prime}}\right) .
$$

At $\Delta^{\prime} \rightarrow \infty$ this reduces to Eq. (10) while at small $\Delta^{\prime}$, neglecting the left-hand side, it reduces to Eq. (11).

\section{Parallel electric field $E_{\|}$}

From Eq. (B6), the parallel electric field is given by

$$
E_{\|}=-i k_{\|}^{\prime}(x \phi+A)=-i k_{\|}^{\prime} \frac{k_{y}^{2} \rho_{s e}^{2} \gamma_{A}^{2}}{\gamma^{2}}\left(\delta_{e}^{2}+x^{2}\right) A^{\prime \prime} .
$$

In the inner zone $x \ll \rho_{s}$, the approximate solution for $A^{\prime \prime}$ is given by Eq. (B15),

$$
E_{\|} \simeq-i k_{\|}^{\prime}\left(\frac{\delta_{e}^{2}+x^{2}}{\delta^{2}+x^{2}}\right) \frac{A_{0}}{1+T_{i} / T_{e}}=E_{\|}(0)\left(\frac{\delta^{2}+\frac{T_{e}}{T_{e}+T_{i}} x^{2}}{\delta^{2}+x^{2}}\right) \text {. }
$$

In the outer zone $x \sim \rho_{s}$, one may use Eq. (B11) to express $A^{\prime \prime}$ in terms of $\phi_{i}^{\prime \prime}$, and then use the expression for $\phi_{i}$ given by Eq. (B18),

$$
\begin{aligned}
E_{\|} & =-i k_{\|}^{\prime} x\left(\frac{\delta_{e}^{2}+x^{2}}{x^{2}}\right) \rho_{s e}^{2} \phi_{i}^{\prime \prime} \\
& \simeq i k_{\|}^{\prime} x\left(\frac{\delta^{2}+\frac{T_{e}}{T_{e}+T_{i}} x^{2}}{x^{2}}\right) \phi_{\infty} e^{-x / \rho_{s}} .
\end{aligned}
$$

\section{Ion velocity stream function $\phi_{i}$}

Here we show the variable $\phi_{i}$ as defined by Eq. (B10) is proportional to the ion velocity stream function in two fluid theory. With this interpretation Eq. (B11) becomes, both physically and mathematically, the ion vorticity equation of the fluid model. Since Eqs. (B10) and (B11) were constructed to be accurate (to leading order) for $\rho_{i}^{2} \nabla_{\perp}^{2} \gg 1$ as well as $\rho_{i}^{2} \nabla_{\perp}^{2} \rightarrow 0$, this accounts for why the two-fluid model yields a correct representation of ion FLR effects in the former limit.

Operating on the ion momentum equation with $\vec{B} \times$, assuming an isothermal ion equation of state, and considering linear perturbations yields

$$
\vec{v}_{i \perp} \simeq \frac{c}{B} \hat{z} \times \nabla_{\perp} \Phi+\frac{1}{\Omega_{c i}} \frac{\partial}{\partial t} \hat{z} \times \vec{v}_{i \perp},
$$

where

$$
\Phi=\phi+\frac{T_{i}}{n_{0} e} \tilde{n} .
$$

Here $\Phi$, aside from a constant factor of $c / B$, is the ion velocity stream function and with Eq. (B10) we will show in a moment that $\Phi=\phi_{i}$. For $\partial / \partial t \ll \Omega_{c i}$ the second term on the right-hand side of Eq. (B23) is small compared to the first, leading to the iterative solution

$$
\vec{v}_{i \perp} \simeq \frac{c}{B}\left(\hat{z} \times \nabla_{\perp} \Phi-\frac{1}{\Omega_{c i}} \frac{\partial}{\partial t} \nabla_{\perp} \Phi\right) .
$$

Substituting this into the linearized ion continuity equation and neglecting the parallel ion motion yields

$$
\frac{\partial}{\partial t} \widetilde{n} \simeq n_{0} \nabla_{\perp} \cdot \vec{v}_{i \perp}=\frac{n_{0} c}{B \Omega_{c i}} \frac{\partial}{\partial t} \nabla_{\perp}^{2} \Phi
$$

or

$$
\tilde{n}=\frac{n_{0} c}{B \Omega_{c i}} \nabla_{\perp}^{2} \Phi .
$$

Substituting this into Eq. (B24) yields $\Phi=\phi+\rho_{i}^{2} \nabla_{\perp}^{2} \Phi$ or equivalently,

$$
\phi=\Phi-\rho_{i}^{2} \nabla_{\perp}^{2} \Phi .
$$

Comparing this to Eq. (B10) one sees that $\Phi=\phi_{i}$ as claimed.

\section{Electron drift kinetic treatment}

The electrons satisfy the drift kinetic equation,

$$
\frac{d}{d t} \tilde{f}+\frac{c}{B \Omega_{c e}} f_{0} \frac{d}{d t} \nabla_{\perp}^{2} \phi=\frac{e}{m_{e}} \frac{\partial f_{0}}{\partial v_{\|}} E_{\|}, \quad \frac{d}{d t}=\frac{\partial}{\partial t}+v_{\|} \nabla_{\|},
$$

where $f=f_{0}+\tilde{f}, E_{\|}=-\nabla_{\|} \phi-\partial A_{z} / \partial t$, and the second term arises from the polarization drift,

$$
\nabla_{\perp} \cdot \vec{v}_{p} f \simeq \frac{c}{B \Omega_{c e}} f_{0} \frac{d}{d t} \nabla_{\perp}^{2} \phi .
$$

Defining $\rho_{e}=v_{\text {the }} / \Omega_{c e}, v_{t h e}=\sqrt{T_{e} / m_{e}}, \Omega_{c e}=e B /\left(m_{e} c\right)$, assuming

$$
f_{0} \simeq \frac{n_{0}}{(2 \pi)^{3 / 2} v_{\text {the }}^{3}} \exp \left[-\left(v_{\perp}^{2}+v_{\|}^{2}\right) /\left(2 v_{\text {the }}^{2}\right)\right]
$$

and taking for linear perturbations $\nabla_{\|}=i k_{\|}^{\prime} x, \partial / \partial t=\gamma$, Eq. (B29) yields

$$
\tilde{f}=-\frac{e f_{0}}{T_{e}} \frac{v_{\|}}{\gamma+i k_{\|}^{\prime} x v_{\|}} E_{\|}-\frac{c f_{0}}{B \Omega_{c e}} \nabla_{\perp}^{2} \phi .
$$

With this expression, the electron density may be written as

$$
\tilde{n_{e}}=\int d^{3} v \tilde{f}=-(1-I) \frac{e n_{0}}{T_{e}} \frac{E_{\|}}{i k_{\|}^{\prime} x}-\frac{e n_{0}}{T_{e}} \rho_{e}^{2} \nabla^{2} \phi,
$$

where

$$
\begin{aligned}
I & =\frac{1}{\sqrt{2 \pi} v_{\text {the }}} \int_{-\infty}^{\infty} d v_{\|} \frac{e^{-v_{\|}^{2} /\left(2 v_{\text {the }}^{2}\right)}}{1+i k_{\|}^{\prime} x v_{\|} / \gamma} \\
& =\frac{2}{\sqrt{\pi}} \int_{0}^{\infty} d z \frac{e^{-z^{2}}}{1+\alpha^{2} z^{2}}, \quad \alpha=\sqrt{2} \frac{x}{\delta_{e}} .
\end{aligned}
$$

Noting that $I \simeq \sqrt{\pi} / \alpha$ for $\alpha \rightarrow \infty$ and $I \simeq 1-\alpha^{2} / 2$ for $\alpha \rightarrow 0$, one is led to the Padé approximation for $1-I$, 


$$
1-I \simeq \frac{x^{2}}{x^{2}+\delta_{e}^{2}}
$$

with which Eq. (B33) can be written as

$$
\tilde{n_{e}}=-\frac{e n_{0}}{T_{e}}\left(\frac{x^{2}}{x^{2}+\delta_{e}^{2}} \frac{E_{\|}}{i k_{\|}^{\prime} x}+\rho_{e}^{2} \nabla^{2} \phi\right) .
$$

If one neglects the $\rho_{e}^{2}$ term on the right-hand side, this equation becomes identical to Ohm's law, Eq. (B5), if one expresses $\widetilde{J}_{\|}$on the left-hand side of Eq. (B5) in terms of $\tilde{n_{e}}$ using Eq. (B2). Continuing, setting $\tilde{n_{i}}=\tilde{n_{e}}$ with $\tilde{n_{i}}$ given by Eq. (B1) one finds

$$
\left(\frac{x^{2}}{x^{2}+\delta_{e}^{2}}\right)(x \phi+A)=\frac{T_{e}}{T_{i}}\left[\Gamma_{0}-1\right] \phi+\rho_{e}^{2} \phi^{\prime \prime}
$$

or using Eq. (B9) to eliminate $\phi$ and rearranging terms,

$$
x(x \phi+A)=\left(x^{2}+\delta^{2}\right) \rho_{s}^{2} \phi_{i}^{\prime \prime}-\rho_{e}^{2} \rho_{i}^{2}\left(x^{2}+\delta_{e}^{2}\right) \phi_{i}^{\prime \prime \prime \prime} .
$$

If one neglects the electron polarization drift term $\propto \rho_{e}^{2}$ on the right-hand side and uses Eq. (B11), this equation reduces to Eq. (B12). Estimating $\phi_{i}^{\prime \prime \prime \prime} \sim \phi_{i}^{\prime \prime} / \delta^{2}$ (valid if the $\rho_{e}^{2}$ term is small) one sees that the ratio of the $\phi_{i}^{\prime \prime \prime \prime}$ term to the $\phi_{i}^{\prime \prime}$ term in Eq. (B38) for $x \sim \delta$ is $\left(T_{i} / T_{e}\right) \rho_{e}^{2} / \delta^{2}$. The same conclusion follows from Eq. (B37), neglecting $\Gamma_{0}$ under the assumption $\delta \ll \rho_{i}$.

${ }^{1}$ M. Kotschenreuther, G. Rewoldt, and W. M. Tang, Comput. Phys. Commun. 88, 128 (1995).

${ }^{2}$ W. Dorland, F. Jenko, M. Kotschenreuther, and B. N. Rogers, Phys. Rev. Lett. 85, 5579 (2000).

${ }^{3}$ E. A. Frieman and L. Chen, Phys. Fluids 25, 502 (1982).
${ }^{4}$ M. A. Beer, S. C. Cowley, and G. W. Hammett, Phys. Plasmas 2, 2687 (1995).

${ }^{5}$ J. Birn, R. E. Denton, J. F. Drake, B. N. Rogers, M. A. Shay, M. Hesse, M. Kuznetsova, Z. W. Ma, and A. Bhattachargee, J. Geophys. Res. 106, 3715, DOI: 10.1029/1999JA900449 (2001).

${ }^{6}$ R. Sydora, Phys. Plasmas 9, 1929 (2001).

${ }^{7}$ W. Wan, Y. Chen, and S. Parker, IEEE Trans. Plasma Sci. 33, 609 (2005).

${ }^{8}$ W. Wan, Y. Chen, and S. Parker, Phys. Plasmas 12, 012311 (2005).

${ }^{9}$ H. Furth, J. Killeen, and M. Rosenbluth, Phys. Fluids 6, 459 (1963).

${ }^{10}$ F. Porcelli, Phys. Rev. Lett. 66, 425 (1991).

${ }^{11}$ J. Drake, Phys. Fluids 21, 1777 (1978).

${ }^{12}$ R. Fitzpatrick and F. Porcelli, Phys. Plasmas 14, 049902 (2007).

${ }^{13}$ D. Grasso, M. Ottaviani, and F. Porcelli, Nucl. Fusion 42, 1067 (2002).

${ }^{14}$ G. Laval, R. Pellat, and M. Vuillemin, Plasma Physics and Controlled Nuclear Fusion Research (IAEA, Vienna, 1965), Vol. II, p. 259.

${ }^{15}$ J. Drake and Y. Lee, Phys. Fluids 2, 1341 (1977).

${ }^{16}$ V. Mirnov, C. C. Hegna, and S. Prager, Phys. Plasmas 11, 4468 (2004).

${ }^{17}$ R. Fitzpatrick and F. Porcelli, Phys. Plasmas 11, 4713 (2004).

${ }^{18}$ J. Drake and M. Shay, "The fundamentals of collisionless reconnection" in Reconnection of Magnetic Fields: Magnetohydrodynamic and Collisionless Theory and Observations, edited by J. Birn and E. R. Priest (Cambridge University Press, Cambridge, 2007), Chap. 3.1.

${ }^{19}$ A. Y. Aydemir, Phys. Fluids 27, 2877 (1981).

${ }^{20}$ L. Zakharov and B. Rogers, Phys. Fluids B 4, 3285 (1992).

${ }^{21}$ B. N. Rogers, R. E. Denton, J. F. Drake, and M. A. Shay, Phys. Rev. Lett. 87, 195004 (2001).

${ }^{22}$ P. Ricci, J. Brackbill, W. Daughton, and G. Lapenta, Phys. Plasmas 11, 4102 (2004).

${ }^{23}$ P. L. Prichett, Phys. Plasmas 12, 062301 (2005).

${ }^{24}$ P. Ricci, J. Brackbill, W. Daughton, and G. Lapenta, Phys. Plasmas 11, 4489 (2004).

${ }^{25}$ B. N. Rogers, R. E. Denton, and J. F. Drake, J. Geophys. Res. 108, 1111, DOI:10.1029/2002JA009699 (2003).

${ }^{26}$ D. Biskamp, R. Sagdeev, and K. Shindler, Cosm. Electrodyn. 1, 297 (1970).

${ }^{27}$ J. Drake and Y. Lee, Phys. Rev. Lett. 39, 453 (1977).

${ }^{28}$ M. Kuznetsova and L. Zelenyi, Plasma Phys. Controlled Fusion 32, 1183 (1990). 